\title{
Télescope
}

Revue d'analyse comparée en administration publique

\section{De la société de l'audit au système des professions : l'histoire institutionnelle de l'audit dans le service public canadien}

\section{Steve Troupin}

Volume 18, numéro 3, automne 2012

URI : https://id.erudit.org/iderudit/1013775ar

DOI : https://doi.org/10.7202/1013775ar

Aller au sommaire du numéro

Éditeur(s)

L’Observatoire de l'administration publique

ISSN

1203-3294 (imprimé)

1929-3348 (numérique)

Découvrir la revue

Citer cet article

Troupin, S. (2012). De la société de l'audit au système des professions : l'histoire institutionnelle de l'audit dans le service public canadien. Télescope, 18(3),

53-71. https://doi.org/10.7202/1013775ar
Résumé de l'article

Si elle permet d'expliquer la croissance de l'audit dans le secteur public, la théorie de la société de l'audit de Michael Power échoue à expliquer pourquoi l'audit, quelquefois, se rétracte. En généralisant les mécanismes de conquêtes professionnelles mises en lumière par Michael Power et en mettant en évidence la compétition interprofessionnelle, la théorie du système des professions d'Andrew Abbott permet de surmonter cette difficulté. L'histoire institutionnelle de l'audit dans le secteur public fédéral canadien est revisitée à l'aide de cette théorie. Elle permet une approche dépassionnée de l'histoire et laisse entrevoir à terme un rééquilibrage des forces professionnelles entre le Bureau du vérificateur général, le Secrétariat du Conseil du Trésor et le Bureau du contrôleur général. 


\section{DE LA SOCIÉTÉ DE L'AUDIT AU SYSTÈME DES PROFESSIONS : L'HISTOIRE INSTITUTIONNELLE DE L'AUDIT DANS LE SERVICE PUBLIC CANADIEN}

Par Steve Troupin, Chercheur, Public Management Institute, KU Leuven, Belgique

- steve.troupin@soc.kuleuven.be

RÉSUMÉ Si elle permet d'expliquer la croissance de l'audit dans le secteur public, la théorie de la société de l'audit de Michael Power échoue à expliquer pourquoi l'audit, quelquefois, se rétracte. En généralisant les mécanismes de conquêtes professionnelles mises en lumière par Michael Power et en mettant en évidence la compétition interprofessionnelle, la théorie du système des professions d'Andrew Abbott permet de surmonter cette difficulté. L'histoire institutionnelle de l'audit dans le secteur public fédéral canadien est revisitée à l'aide de cette théorie. Elle permet une approche dépassionnée de l'histoire et laisse entrevoir à terme un rééquilibrage des forces professionnelles entre le Bureau du vérificateur général, le Secrétariat du Conseil du Trésor et le Bureau du contrôleur général.

ABSTRACT While Michael Power's theory of the "audit society" is useful for explaining the expansion of auditing in the public sector, it nevertheless fails to explain why auditing practice occasionally contracts. This difficulty can be surmounted, however, through Andrew Abbott's "system of professions" theory, which generalizes the mechanisms of professional turf battles brought out by Michael Power and which highlights interprofessional competition. This theory is used to review the institutional history of auditing in the Canadian federal public sector. It is of greater use in developing a detached approach to history and suggests that in the long run, rebalancing will occur between the Office of the Auditor General, the Treasury Board of Canada Secretariat, and the Office of the Comptroller General.

Pour citer cet article : Troupin, S. (2012). « De la société de l'audit au système des professions : I'histoire institutionnelle de l'audit dans le service public canadien », Télescope, vol. $18, n^{\circ} 3$, p. 53-71.

ette contribution examine les explications possibles à la croissance de l'audit dans le service public fédéral canadien. L'audit y a en effet connu un démarrage spectaculaire entre 1960 et 1980, avant de reculer progressivement jusqu'en 1994 et de reprendre de plus belle récemment, dans la foulée du scandale des commandites. L'explication la plus évidente - et celle qui est de loin la plus répandue - lie l'émergence de l'audit aux fonctions qu'il remplit : la croissance de l'audit répond à une demande grandissante des pouvoirs publics pour une information objective. En somme, l'audit est là parce que nous en avons besoin. Toutefois, les observateurs attentifs de la vie politique canadienne objecteront que le gouvernement était loin d'être demandeur, dans les années 1970, d'une telle croissance de l'audit.

Dans la théorie de la société de l'audit, Michael Power (1999) renverse l'explication en examinant comment l'essor de l'audit répond à une fonction du point de vue des auditeurs. Si on analyse cette contribution de façon cumulative, une 
seconde cause apparaît : l'audit progresse dans le secteur public parce que les auditeurs prétendent pouvoir remplir la demande politique pour une fonction particulière. Bien qu'elle constitue un progrès, cette théorie n'est toutefois pas en mesure d'expliquer le retrait temporaire de l'audit au Canada autour de 1993 : le maintien des prétentions de la profession n'a pas permis d'éviter ce recul.

Dans cet article, je soutiens que la théorie du système des professions (system of professions) d'Andrew Abbott (1988) permet de perfectionner cette explication de la croissance et du recul de l'audit et d'autres pratiques professionnelles dans le secteur public. Abbott ajouterait une troisième cause : l'audit croît dans le secteur public parce que les auditeurs sont mieux parvenus que leurs concurrents à prétendre pouvoir remplir la demande politique pour une fonction particulière :

Les professions constituent une écologie. [...] Elles souhaitent s'agrandir en compétition, prenant le pouvoir sur cette aire-ci de travail ou celle-là, qu'elles transforment en " juridiction " au moyen des systèmes professionnels de connaissance. Une variété de forces internes et externes créent perpétuellement des possibilités de gain ou de perte de juridictions. Les professions pro-agissent et réagissent en saisissant ces occasions et en renforçant ou en abandonnant leurs juridictions précédentes. À côté de cette constitution symbolique de tâches en juridictions construites, identifiées, les différents instruments structurels de la professionnalisation - devenant tantôt plus forts, tantôt plus faibles - fournissent un point d'ancrage structurel aux professions. De plus, chaque événement juridictionnel qui arrive à une profession mène les professions adjacentes à de nouvelles ouvertures ou défaites (Abbott, 2005, p. 246).

Telle est, dans sa version la plus succincte, la théorie du système des professions à travers laquelle j'analyse l'histoire de l'institutionnalisation de l'audit dans le secteur public fédéral canadien. À cette fin, je considère des organisations, le Bureau du vérificateur général (BVG), le Bureau du contrôleur général (BCG), le Secrétariat du Conseil du Trésor (SCT) et le ministère des Finances, comme des groupes professionnels au sens d'Abbott, en mettant en évidence la plus-value de cette théorie par rapport à celle de Michael Power. Les données ont été recueillies au cours d'une analyse systématique de la littérature scientifique et organisationnelle et, en ce qui concerne les cas les plus récents, sur la base d'une série d'interviews menées à Ottawa à l'automne 2009 avec les principales parties prenantes de l'audit dans le gouvernement fédéral canadien ${ }^{1}$.

J'examine d'abord la théorie de la société de l'audit de Michael Power puis celle du système des professions d'Andrew Abbott. Je revisite ensuite les différents épisodes de la croissance et du retrait de l'audit dans le gouvernement fédéral canadien, en mettant en évidence les apports de la théorie d'Abbott par rapport à celle de Michael Power. Enfin, je discute de quelques leçons que cette théorie du système des professions nous permet de tirer quant à l'évolution de l'audit dans le gouvernement canadien et à la professionnalisation des organisations publiques plus généralement.

1 Ces interviews ont été menées avec des cadres ministériels du Bureau du Conseil privé, du Bureau du vérificateur général, du Bureau du contrôleur général, du Secrétariat du Conseil du Trésor, du Comité permanent des comptes publics et d'un professeur de l'Université d'Ottawa. 


\section{- LA SOCIÉTÉ DE L’AUDIT AU CANADA}

Peut-être en raison de la façon par laquelle le gouvernement fédéral du Canada a été contraint d'attribuer au BVG l'un des premiers mandats au monde de vérification de gestion (Glynn, 1985), la théorie de la société de l'audit de Michael Power qui sera publiée ultérieurement semble profiter d'un large écho dans les milieux universitaires et professionnels. La société de l'audit désigne le nombre de plus en plus important d'activités effectuées selon le savoir abstrait de l'audit. Elle résulte, selon Power (1999), de deux phénomènes.

\section{FIGURE 1: LA THÉORIE DE LA SOCIÉTÉ DE L'AUDIT DE MICHAEL POWER (1999)}

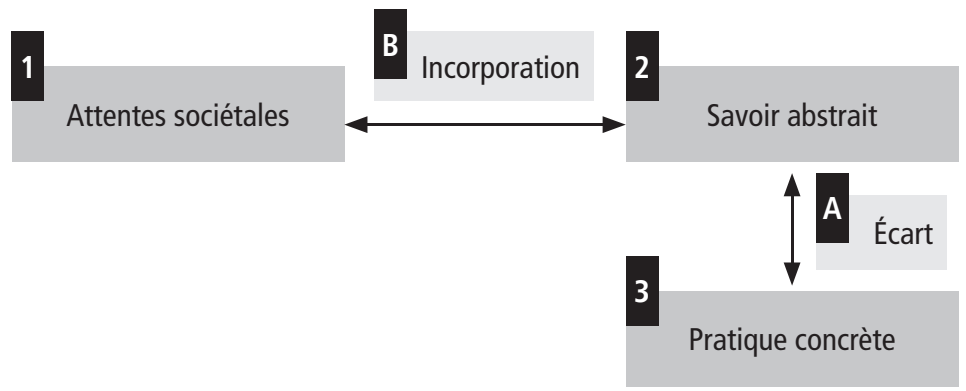

D'une part, Power distingue le savoir abstrait $(2)^{2}$ de la pratique concrète (3) de l'audit. Il soutient que l'audit, en tant que pratique (3), est « fondamentalement obscur ", c'est-à-dire que son caractère fondamentalement subjectif et herméneutique (Francis, 1994) résiste à la formalisation théorique : deux auditeurs vérifiant la même organisation aboutiront vraisemblablement toujours à des conclusions différentes. Dans son savoir abstrait (2), soutient Power, l'audit nie toutefois cette réalité; il est toujours présenté (B) comme une technique neutre et objective afin de se conformer aux attentes que la société place en lui (1). Dès lors, précise-t-il, un écart (A) apparaît entre le savoir abstrait de l'audit (objectif et reproductible) et sa pratique concrète (subjective et idiosyncrasique). D'autre part, lorsque de nouvelles demandes sociétales apparaissent relativement à la fourniture de nouveaux types d'information objective (1), la profession d'audit se propose d'y répondre. À cette fin, elle intègre ces nouvelles exigences dans son savoir abstrait (2), sans toutefois fondamentalement modifier les pratiques concrètes, irréductibles à l'accomplissement successif d'un certain nombre d'étapes (3). En conséquence, soutient Power, l'écart (A) entre le savoir abstrait et la pratique concrète augmente.

\footnotetext{
${ }^{2}$ Les chiffres et les lettres entre parenthèses renvoient à la figure 1.
} 
Comme le caractère " fondamentalement obscur " de l'audit est caché derrière son savoir abstrait, Power affirme qu'aucun non-auditeur n'est en mesure de vérifier l'étendue de cet écart et que la profession d'audit peut dès lors tranquillement poursuivre l'intégration de nouvelles demandes sociétales dans son savoir abstrait, qui s'applique dès lors à un nombre sans cesse croissant de nouvelles activités, menant finalement à une société de l'audit.

L'ouvrage de Power (1999) peut être lu comme une tentative de rendre visible ce décalage entre savoir abstrait et pratique concrète de l'audit afin de permettre à la société de confier de nouvelles fonctions à l'audit selon son potentiel réel limité plutôt que proclamé. La théorie de la société de l'audit peut être considérée comme la théorisation post hoc des événements ayant mené au mandat de verification de gestion du BVG et à la création du BCG à la fin des années 1970. Sharon Sutherland (2002, 1990, 1986, 1981 et 1980) a d'ailleurs invoqué cet argument de façon empirique dans de nombreux travaux. D'une part, elle s'est attachée à démontrer que la technique de vérification de gestion du BVG, bien que présentée comme objective, est "fondamentalement obscure ", subjective, ce qui justifierait la réintégration de ce travail au système démocratique. D'autre part, elle montre comment le BVG est parvenu à intégrer dans son savoir abstrait les idéaux scientifiques ayant présidé aux réformes administratives précédentes afin de soustraire un certain nombre d'activités étatiques au contrôle démocratique pour les confier à son avatar gouvernemental, le BCG.

Le ton général adopté par Sutherland correspond parfaitement à celui de Power : tous deux insistent sur le caractère usurpé des responsabilités confiées aux auditeurs et appellent la société à ouvrir les yeux et à corriger ce qui est présenté implicitement comme une erreur historique.

Toutefois, alors que la théorie de la société de l'audit lit l'histoire comme la progression irrésistible de l'audit vers une situation de monopole absolu, cette société de l'audit canadienne naissante a connu un sérieux revers en 1993 lorsque l'un de ses deux dépositaires institutionnels, le BCG, a cessé d'avoir une existence propre dans l'appareil étatique et que son travail a perdu toute affiliation intellectuelle avec le savoir abstrait de l'audit pour être intégré dans une politique générale de coupes budgétaires.

La théorie de Power n'étant pas en mesure d'expliquer le recul temporaire de l'audit dans le gouvernement du Canada, je propose de relire l'histoire de l'audit dans le gouvernement canadien à l'aide d'une théorie qui le permet, soit celle du système des professions développée par Andrew Abbott (1988) et de voir les nouvelles perspectives que ce type d'analyse nous offre. 


\section{- DE LA SOCIÉTÉ DE L'AUDIT AU SYSTÈME DES PROFESSIONS}

Andrew Abbott (1988) considère qu'il n'existe pas un, mais plusieurs groupes professionnels susceptibles de recourir au mécanisme mis en lumière par Power pour s'approprier ces activités. Cette appropriation, une fois réalisée, est appelée juridiction ${ }^{3}$. Reformulée dans le langage d'Abbott, la société de l'audit signifie que le groupe professionnel des auditeurs possède un nombre sans cesse croissant de juridictions. Abbott $(1988$, p. 8) définit les groupes professionnels comme des groupes appliquant un savoir abstrait à des cas concrets : l'écart entre le caractère " fondamentalement obscur " des pratiques et leur représentation abstraite est, pour Abbott, précisément ce qui distingue les professions des autres groupes occupationnels.

Cet écart permet aux professions d'intégrer dans leur savoir abstrait différents problèmes humains (Abbott, 1988, p. 36-52) et de revendiquer (op. cit., p. 98-101) le droit exclusif de s'en charger à travers différents mécanismes rhétoriques tels que la réduction (reformulation d'un problème comme étant soluble par un savoir abstrait particulier), la métaphore (diffusion d'une façon de penser un problème selon le vocabulaire d'un savoir abstrait), le traitement (revendication de la capacité d'un savoir abstrait de résoudre des problèmes identifiés par d'autres) ou l'argument gradient (revendication de considérer les causes d'un problème plutôt que ses conséquences). Dans cet article, le concept de zone de tâche est utilisé comme synonyme de celui de problème humain, à savoir un travail qui n'a pas encore acquis la définition que lui donnera le savoir abstrait qui s'appliquera sur lui : cette zone de tâche non encore constituée en juridictions et constituant l'objet de l'analyse conduite ici, nous la définissons comme l'analyse a posteriori de la performance de l'administration centrale du gouvernement fédéral; l'enjeu étant de savoir si cette zone de tâche deviendra une juridiction d'audit, de budget, de gestion ou de tout autre savoir.

Les groupes professionnels déposent leurs différentes revendications devant trois auditoires : l'opinion publique, l'État et le lieu de travail. Abbott (1988, p. 6067) insiste sur le décalage entre les règlements juridictionnels sur ces différents auditoires. Par exemple, ce n'est pas parce que le savoir abstrait de l'audit est formellement chargé de régler le problème de la performance administrative que les auditeurs ont un pouvoir correspondant au sein de chaque administration : il pourrait très bien y être réglé par le savoir abstrait de la gestion.

Ces auditoires apprécient les revendications selon leurs propres critères (Abbott, 2005) : alors que l'objectif du groupe professionnel est d'obtenir la res-

\footnotetext{
${ }^{3}$ Le terme « juridiction » traduit le concept anglais jurisdiction. Habituellement utilisé en français pour désigner une cour ou un tribunal, il renvoie ici au pouvoir de juger, pris au sens large : il doit se comprendre comme le droit exclusif octroyé à un groupe de concevoir et d'exercer un travail professionnel donné (Abbott, 1988). Ainsi, il pourrait également être traduit par compétence ou responsabilité. Toutefois, dans la mesure où l'auteur de ce concept l'a traduit par « juridiction » dans un article original en français (Abbott, 2003), et que cet usage s'est perpétué dans de nombreux ouvrages français de sociologie des professions (notamment Dubar et Tripier, 1998; Champy, 2009), cette traduction sera conservé afin d'en indiquer la filiation intellectuelle.
} 
ponsabilité du problème à régler et les ressources matérielles et symboliques qui s'y rattachent, l'auditoire est censé vouloir voir le problème en question résolu. Dès lors qu'une revendication répond aux intérêts d'un auditoire, une juridiction naît : une juridiction est la ratification par un auditoire de la compétence exclusive d'un savoir abstrait et de son dépositaire institutionnel à prendre soin du problème en question. Nous retrouvons ici les deux conditions posées par Michael Power pour la réalisation de la société de l'audit : un savoir abstrait, découplé des pratiques professionnelles, mais aligné sur des demandes politiques, dans le cas de l'auditoire étatique.

Abbott se distingue toutefois fondamentalement de Power en considérant l'interdépendance des groupes professionnels, là où Power dépeint l'imperturbable marche en avant d'une seule profession. Il distingue en effet les situations pour lesquelles la juridiction est vacante, c'est-à-dire celles où aucun autre groupe ne se charge déjà d'aborder le problème dont il est question, de celles où la juridiction est occupée par un autre groupe concurrent. Dans le premier cas, les mécanismes mis en lumière par Power peuvent opérer. Dans l'autre cas, nous explique-t-il, des conditions supplémentaires doivent être remplies afin que s'opère un transfert juridictionnel. D'une part, le groupe revendicateur doit être enclin à déloger son concurrent (Abbott, 1988, p. 95-97). C'est le cas lorsqu'un nouveau groupe est créé, lorsqu'un groupe existant perd des juridictions ou lorsqu'il a construit son savoir abstrait de façon à entrevoir de nouvelles applications possibles. D'autre part, le groupe occupant doit détenir sa juridiction faiblement (Abbott, 1988, p. 96-103), c'est-à-dire qu'il doit être perçu comme ayant échoué à résoudre le problème dont il avait la charge (Champy, 2009). Contrairement à Abbott, Power n'a pas estimé que la présence d'autres groupes puisse constituer un obstacle au déploiement de la société de l'audit.

Enfin, à l'inverse de Power qui considère potentiellement comme infini l'écart entre le savoir abstrait et les pratiques concrètes, Abbott (1988, p. 103-108) y pose une limite. Le savoir abstrait d'une profession est le plus petit dénominateur commun de ses juridictions : au plus l'abstraction de l'audit doit s'appliquer à un grand nombre de situations, au plus il doit être défini de façon large. Dès lors qu'un savoir trop abstrait (risque de déconnexion du travail réel) ou qu'un savoir trop concret (risque de marchandisation du savoir - Abbott, 1988, p. 146) est susceptible de permettre à un concurrent d'établir l'échec de l'occupant et de le déloger, il soutient que les groupes professionnels cherchent à se neutraliser à long terme, pour tendre vers une situation d'équilibre dans laquelle chaque groupe professionnel détient une seule juridiction qu'il est en mesure d'effectuer convenablement. Power, en mettant en avant la marche ininterrompue de la profession d'audit vers la domination et le caractère trop abstrait de son savoir, amorce en réalité un retour de flamme.

L'hypothèse selon laquelle la théorie du système des professions explique mieux l'émergence de la société de l'audit canadienne que celle de Power est justifiée par l'incapacité de cette dernière de justifier le recul de 1993. Elle ouvre des perspectives plus larges. Plutôt que de confirmer formellement la pertinence de cette hypothèse - ce que Troupin (2012) fait par ailleurs -, je propose de passer 
brièvement en revue l'histoire des heurs et des malheurs de la société de l'audit canadienne par le truchement de cette théorie, afin de mettre en lumière les nouvelles leçons qu'elle permet d'en tirer.

Les luttes juridictionnelles formant le pivot de la théorie d'Abbott, il est possible de découper l'histoire de l'audit dans le gouvernement fédéral canadien en cinq cas correspondant à autant de luttes, remportées ou non : l'obtention par le BVG d'une juridiction de vérification de gestion, le transfert de juridictions relatives à l'organisation de la production ministérielle d'analyses de la performance du SCT vers le BVG, l'échec du BVG à absorber le BCG, la réintégration de ce dernier et des juridictions au sein du SCT et, enfin, son rétablissement à la suite du scandale des commandites.

\section{- LE SECTEUR PUBLIC CANADIEN EN TANT QUE SYSTÈME DES PROFESSIONS}

\section{Le Bureau du vérificateur général du Canada n'a pas usurpé sa juridiction de vérification de gestion}

L'analyse opérée par Sutherland (2002, 1986, 1981 et 1980) à propos de la façon par laquelle le BVG a obtenu sa juridiction de vérification de gestion correspond à la théorie de la société de l'audit : comme deux auditeurs appliquant ce savoir abstrait arriveraient à des conclusions différentes, la vérification de gestion est subjective; comme la résolution de conflits subjectifs est le propre du politique, c'est au gouvernement et non au BVG qu'il revient d'analyser la performance administrative. Vu du système des professions par contre, l'enjeu consiste précisément à savoir quel groupe effectuera quel travail et au nom de quels principes. Ce cas s'apparente à l'exercice normal de la compétition professionnelle.

En effet, aux yeux du public, la performance administrative relevait déjà du BVG. En 1951, la Loi sur la gestion des finances publiques autorise le BVG à rapporter au Parlement toute information qui mérite son attention. Sur cette base, le vérificateur général Watson Sellar prend l'habitude dès 1958 de conclure son rapport annuel par une liste de dépenses qu'il estime non productives. Son successeur, Maxwell Henderson, étend considérablement cette pratique, allant jusqu'à rapporter les montants totaux dépensés de façon non productive, pour le plus grand plaisir du Parlement et des médias : à leurs yeux, le professionnel de la performance administrative n'est déjà plus le gouvernement, mais le BVG, et son travail est connu comme Les récits effroyables d'Henderson (Henderson's Horror Stories; Sinclair, 1979, p. 61-75).

Dans les années 1970, le vérificateur général James Macdonnell tire argument de cette juridiction publique pour obtenir une juridiction similaire de l'auditoire étatique. Pour ce faire, il recourt à un argument gradient (Abbott, 1988, p. 101) : dans la mesure où je dois rapporter au Parlement les cas pour lesquels des fonds publics ont été dépensés de façon peu opportune, soutient-il en substance, et considérant que le problème est plus structurel que ponctuel, ne serait-il pas plus approprié d'examiner le fonctionnement de ces structures afin de prévenir de 
telles dépenses plutôt que de se contenter de compter les dégâts (Sinclair, 1979, p. 101-113)? Il réclame donc une juridiction de vérification de gestion.

Le comité Wilson, appelé à statuer sur cette revendication, ne voit d'autre objection que les études d'efficacité : sujettes à controverse politique, elles pourraient mettre en danger l'indépendance du BVG. Toutefois, compte tenu de leur grande importance, il estime opportun d'octroyer au BVG le droit d'analyser les études produites par le gouvernement (Sinclair, 1979, p. 115-125).

Comme l'explique Sinclair (1979, p. 189), le gouvernement n'a alors d'autre choix que d'accepter la revendication du BVG : « Macdonnell allait aller de l'avant avec ses vérifications, avec ou sans [juridiction légale] ; et si quelqu'un formulait une objection, il renverrait aux conclusions du comité Wilson en disant "[...] voudriezvous dire que je ne suis pas censé regarder l'argent du contribuable dépensé de façon extravagante?" Dès lors que la réponse du public à cette question ne faisait aucun doute, le gouvernement peut avoir décidé de céder élégamment plutôt que d'engager une bataille qui n'aurait rien pu faire pour augmenter sa popularité. » Le $1^{\text {er }}$ août 1977, la Loi sur le vérificateur général ratifie cette revendication : le BVG possède une juridiction de vérification de gestion entière en ce qui concerne les vérifications d'économie et d'efficience, et à partager avec le gouvernement en ce qui concerne les études d'efficacité.

En d'autres termes, selon la théorie d'Abbott, le gouvernement avait perdu la main dès 1951, et la traduction de cette juridiction publique dans l'ordre légal n'est que la conclusion d'un long processus historique, et non un coup de force momentané.

\section{Le Bureau du contrôleur général n'aurait jamais existé si la rationalisation des choix budgétaires n'avait échoué}

Dès 1962, la Commission royale d'enquête sur l'organisation du gouvernement (aussi appelée commission Glassco), chargée de trouver des solutions au problème de la performance administrative, formule deux recommandations : libérer les managers des contraintes centrales relatives à la gestion financière et du personnel et remplacer le système budgétaire actuel par la rationalisation des choix budgétaires (Rowat, 1963; Saint-Martin, 1998).

Ces recommandations entraînent la création d'un nouveau groupe professionnel au sein du secteur public canadien, le SCT, qui doit assumer deux responsabilités (Johnson, 1971). En ce qui concerne la rationalisation des choix budgétaires, d'une part, le travail du SCT consiste à proposer une classification des dépenses gouvernementales en trois catégories - programmes poursuivis, nouveaux programmes et programmes à supprimer - en s'appuyant sur les études d'efficacité et d'efficience produites par les ministères sous sa direction (Johnson, 1971). D'autre part, il est du ressort du SCT d'exécuter les décisions budgétaires des ministères. Le SCT ne parviendra toutefois pas à mettre en œuvre ces deux responsabilités de façon satisfaisante. Divers problèmes conceptuels, techniques et politiques rendront impossible l'établissement scientifique de priorités budgétaires (Harder et Lindquist, 1997; Johnson, 2002, p. 301-305; Sutherland, 2002). En outre, 
dans l'esprit de la commission Glassco ("Let the manager manage "), le SCT exerce son autorité relative à l'exécution du budget d'une façon tellement souple qu'elle confine à l'abandon (Johnson, 1971) : la direction responsable n'apparaît pas sur l'organigramme et elle est composée d'une demi-douzaine de fonctionnaires de second rang (Sinclair, 1979, p. 127-130 et 133).

Le BVG intervient en rendant visible cette faible détention juridictionnelle lors de sa fameuse intervention au Parlement en 1976 où il s'estime " profondément inquiet que le Parlement ait perdu ou soit en train de perdre la maîtrise effective des deniers publics ». Par conséquent, il formule une revendication visant à transférer l'essentiel de ces pouvoirs à un nouveau groupe professionnel, le BCG (Sinclair, 1979, p. 143). Selon la théorie d'Abbott, la démonstration de cet échec était nécessaire à la création du BCG. Le gouvernement crée alors la Commission royale sur la gestion financière et l'imputabilité (ou commission Lambert) pour traiter le problème de gestion financière mis à l'ordre du jour par le BVG. Il a espoir qu'elle formule une revendication concurrente à celle du BVG. Las, celle-ci se prononce immédiatement en faveur de la création du BCG (Hodgetts, 2007, p. 531532; O’Neal, 1994 ; Sinclair, 1979, p. 145).

En 1979, le gouvernement semble changer son fusil d'épaule en remplaçant la rationalisation des choix budgétaires par le système de gestion des secteurs de dépenses (Good, 2007, p. 256-258). Ce système prévoit un autre rôle pour le SCT : au lieu de préparer la répartition scientifique de toutes les dépenses en programmes nouveaux, à poursuivre et à supprimer, il lui revient maintenant d'attribuer une petite réserve opérationnelle de façon plus politique que scientifique (Good, 2007, p. 64-68; Savoie, 1996, p. 68).

L'analyse de cet événement par la théorie d'Abbott laisse à penser que le gouvernement aurait pu profiter de la revendication du BVG pour liquider l'encombrant héritage de la rationalisation des choix budgétaires et pour préserver un cœur de métier pour le SCT, un peu à la manière des crédits toxiques des banques hérités de la crise des subprimes qui sont actuellement transférés dans des bad banks - une hypothèse toujours pas confirmée par la littérature. Le système de gestion des secteurs de dépenses ne prévoyant de fonder des décisions budgétaires sur la science que de façon supplétive (Good, 2007, p. 62), il a pu paraître opportun de laisser le BVG se débrouiller avec son avatar gouvernemental. Toujours est-il que le BCG est créé en 1978 et que le gouvernement lui confie, outre la gestion financière, l'organisation de la production des études d'efficience et d'efficacité, l'analyse de ces dernières relevant de la vérification de gestion du BVG.

La théorie d'Abbott nous permet de nuancer l'analyse de cet épisode en termes de hold-up de la part du BVG : la revendication publique du BVG n'était qu'une condition parmi d'autres à la création du BCG. D'une part, comme le travail considéré était exercé par le SCT, il fallait que ce dernier échoue et que cet échec soit rendu visible : jamais le BCG ne serait apparu si le SCT n'avait abandonné sa juridiction relative à l'exécution du budget et si le BVG n'avait rendu cela visible. D'autre part, il peut être soutenu que le gouvernement a eu quelque intérêt à satisfaire la revendication du BVG : cela permettait de découpler la gestion quotidienne du gouvernement de la production d'études de performance. 


\section{Il n'y a pas de société de l'audit mais un système des professions}

Concomitamment à ces deux épisodes, le BVG met en place les conditions de la fusion des deux groupes professionnels au sein d'une société de l'audit.

Premièrement, le BVG profite de sa division du travail avec le BCG relativement aux études d'efficacité (le BCG est chargé d'organiser la production de ces études, alors que le BVG doit les analyser) pour réguler indirectement le travail du BCG. Le projet SPICE (Study of Procedures in Cost Effectiveness) lui permet d'ailleurs d'affirmer qu'il existe une méthode objective pour analyser l'efficacité des programmes publics (Sutherland, 1980, p. 629-637) et il invite le BCG à exercer son travail selon ce savoir abstrait qu'il conçoit. Deuxièmement, le BVG entreprend de fusionner ses tâches de vérification financière, de vérification de gestion et d'analyse des études d'efficacité en une seule compétence de vérification intégrée (Cutt, 1988; Sinclair, 1979, p. 195-196; Sutherland, 1980, p. 621). Indirectement, en mettant ce savoir à la disposition du BCG nouvellement créé et équipé de juridictions parallèles, il l'encourage à effectuer son travail selon la façon dont il se le représente. Enfin, en plus des structures intellectuelles, le BVG crée les structures organisationnelles nécessaires à la fusion des deux groupes en un groupe professionnel de vérificateurs intégrés par l'entremise de la Fondation canadienne pour la vérification intégrée (Sutherland, 2002, p. 13).

Il ne manquait plus au BVG que le consentement du BCG afin de finaliser la société de l'audit. Harry Rogers, premier contrôleur général, refuse toutefois cette invitation. Tout d'abord, là où le BVG fusionne ses compétences, il en distingue trois dans la même zone de tâche : la gestion financière, la mesure des performances et l'évaluation des programmes (Rogers, 1978, p. 17). Ensuite, il rejette l'existence d'une méthodologie parfaitement objective en matière de vérification, soutenant que deux analyses effectuées selon la même méthode aboutiraient à deux résultats différents (Rogers, cité dans Sutherland, 1980, p. 638). Enfin, le BCG ne figurera jamais sur la liste des membres de la Fondation canadienne pour la vérification intégrée.

Cet épisode permet une fois de plus de nuancer sérieusement la thèse de la société de l'audit. Premièrement, il semblerait qu'il n'y ait pas un, mais bien plusieurs auditeurs. Deuxièmement, la filiation intellectuelle commune de ces deux groupes n'implique pas leur alliance dans une société de l'audit : l'impérialisme intellectuel du BVG n'a pas suffi à rallier le BCG à son projet professionnel (Larson, 1977); il faut être deux pour danser le tango. Troisièmement, le gouvernement n'a pas été cet oiseau pour le chat que les tenants de la thèse de la société de l'audit aiment à dépeindre : bon an mal an, il est parvenu à développer une revendication concurrente et à limiter les dégâts pour le SCT. Plus généralement, il semble qu'aucun groupe ne soit en mesure d'imposer définitivement sa volonté à l'autre.

\section{De l'exclusivité du travail professionnel}

Alors que la théorie de la société de l'audit ne connaît pas de limites à son expansion, celle d'Abbott assume la prévalence d'un équilibre à plus long terme. Les événements ayant mené à la réintégration en 1993 du BCG au sein du SCT semblent lui donner raison. 
L'échec de la rationalisation des choix budgétaires a laissé le SCT fort diminué : l'essentiel de son travail ayant été redistribué entre le ministère des Finances et le BCG, il ne lui reste plus, sous le système de gestion des secteurs de dépenses, qu'une petite juridiction relative à l'allocation d'une réserve opérationnelle. Toutefois, trois facteurs vont contribuer à lui enlever cette responsabilité également. Premièrement, une nouvelle juridiction apparaît concomitamment au système de gestion des secteurs de dépenses, que Savoie (2008) appellera plus tard "Strategic Prime Ministership ", et qui consiste à extraire certaines zones de tâche de leur champ d'application formelle pour les faire relever du premier ministre. À partir de 1984, l'extension continue de cette juridiction a pour effet de diminuer progressivement l'objet du travail du SCT et d'autres acteurs (Johnson, 2002, p. 195-198). Aussi, la compétence du SCT sur la réserve opérationnelle disparaît progressivement avec celle-ci, à mesure que le ministère des Finances diminue sa taille sous l'effet de la crise budgétaire approchante (Good, 2007, p. 67; Kelly et Lindquist, 2003). Enfin, une série de réformes administratives aboutit à déléguer aux cadres ministériels un nombre croissant de responsabilités, diminuant leur dépendance de la réserve opérationnelle du SCT (Good, 2007, p. 263; O’Neal, 1994; Paquin, 1997). Selon Abbott (1988), au début des années 1990 le SCT se trouve dans une situation de déficit juridictionnel : la perte de ce travail professionnel l'incite naturellement à en gagner d'autres en délogeant des concurrents.

Pendant ce temps, le BCG échoue à mettre en œuvre ses compétences de façon satisfaisante. Premièrement, une certaine confusion semble s'installer tant au BCG qu'au sein des ministères quant au contenu réel du travail. Le BCG, après avoir refusé de fusionner ses juridictions dans un contrôle compréhensif, distingue en 1978 administration financière, mesure des performances et évaluation des programmes (Rogers, 1978). Deux ans plus tard, l'administration financière devient la vérification interne, qui se partage avec l'évaluation des programmes ce qui était jusque-là la mesure des performances (Brophy et McGimpsey, 1980). Cette confusion se reproduit sur le lieu de travail, où les deux juridictions sont fréquemment fusionnées dans une division de revue des programmes (OAG, 1987) puis séparées à nouveau (Brophy et McGimpsey, 1980). Deuxièmement, la formalisation du travail professionnel des différentes juridictions décline dès 1982 pour finalement être arrêtée en 1986 (Free et Radcliffe, 2009; Sutherland, 1990). Troisièmement, l'exécution pragmatique de l'évaluation des programmes semble remettre en question la nature professionnelle du travail (Sutherland, 1990). Enfin, le personnel qualifié pour effectuer le travail dans les ministères manque et les différentes compétences se chevauchent largement sur le lieu de travail (Hollander et Prince, 1993; Prince et Chenier, 1980) : le BCG est dans une situation qu'Abbott qualifierait d'excès juridictionnel (Abbott, 1988, p. 257). Dans ses nombreux rapports sur son avatar gouvernemental, le BVG (OAG, 1983, 1984, 1987 et 1993) rend visible cet échec progressif du BCG.

En 1993, ces deux événements se réunissent : le SCT réintègre le BCG et ses compétences dans ce qui semble être une résurrection de la rationalisation des choix budgétaires. Dans un contexte de crise budgétaire, le SCT est en effet censé générer des économies sur la base des études produites par les ministères (O'Neal, 
1994). Toutefois, cette politique de réduction scientifique des dépenses dégénère immédiatement en un exercice d'attribution arbitraire de réduction de dépenses, sous la puissante impulsion du ministère des Finances (Good, 2007, p. 267-268; Johnson, 2002, p. 369). La rupture du lien entre études et coupes budgétaires laisse le SCT avec des responsabilités assez semblables à celles du BCG qu'il vient d'absorber. En conséquence, il se redéfinit comme le conseil de gestion du gouvernement détenant une juridiction générale de gestion.

\section{De la gestion à la vérification interne}

Le BVG est évidemment furieux du sort réservé à son avatar gouvernemental et réclame la délimitation d'un contrôleur général et d'une juridiction associée à l'intérieur du SCT (OAG, 1993). Le SCT mandate alors le Groupe de travail indépendant chargé de la modernisation de la fonction de contrôleur dans l'Administration fédérale du Canada, dirigé par le président de la Fondation canadienne pour la vérification intégrée, pour développer un savoir abstrait pour soutenir son autorité de gestion, et celui-ci propose exactement la même chose que le BVG. La politique de vérification interne du SCT de 2001 ratifie cette conception : à l'intérieur du champ de gestion, la juridiction de vérification interne est attribuée à un BCG qui possède formellement vis-à-vis du SCT un statut professionnel similaire à celui des psychiatres vis-à-vis des médecins : celui d'un groupe intellectuellement autonome et structurellement fédéré (Abbott, 1988, p. 105-108).

Malheureusement, cette nouvelle architecture n'est pas confirmée sur le lieu de travail : les différentes versions du concept de gestion (y compris la vérification interne) se chevauchent et entrent en concurrence pour l'obtention d'une juridiction sur le lieu de travail (Kelly et Lindquist, 2003). Le regard que portent Clark et Swain (2005), deux cadres ministériels, sur les « exigences surréalistes » du SCT et la façon dont ils se proposent de les prendre en compte (en les isolant de la vie organisationnelle réelle) illustrent parfaitement la faible détention de compétence du SCT et du BCG.

L'éclatement en 2002 du scandale des commandites, impliquant la rétribution de bailleurs de fonds privés du Parti libéral au pouvoir par l'intermédiaire de programmes publics, mène à différentes investigations. D'une part, le BVG (OAG, 2002), en indiquant que " les hauts fonctionnaires ont enfreint toutes les règles ", montre l'urgence de s'attaquer aux problèmes sans toutefois préciser comment les résoudre, si ce n'est par la publication concomitante d'un rapport mettant en lumière l'échec du SCT-BCG à traduire sur le lieu de travail la compétence de vérification interne. D'autre part, la commission d'enquête Gomery chargée de faire la lumière sur ce scandale publie différents rapports, dont un semble indiquer que le scandale aurait pu être évité si les vérificateurs internes ne s'étaient pas autocensurés (Benoit et Franks, 2006). En réaction, le gouvernement entreprend d'extraire le BCG du SCT avec ses juridictions relatives à la vérification interne d'abord, et du contrôle interne en matière financière ensuite (Free et Radcliffe, 2009).

Cet épisode a également des conséquences pour le BVG. D'une part, pour la première fois de son existence, il a été obligé d'exposer devant la commission d'enquête la façon par laquelle il est parvenu à élaborer ce qui deviendra la vérité 
officielle sur ce scandale (Fraser, 2007). Le BVG a passé son examen avec brio (Fraser, 2004) bien qu'il s'en soit fallu de peu pour que l'écart entre son savoir abstrait et ses pratiques concrètes n'apparaisse au grand jour (interview avec un cadre du BVG, 2009). D’autre part, la série de mesures adoptées par le gouvernement afin de communiquer sa bonne volonté de prévenir l'apparition ultérieure de scandales similaires semble attribuer au BVG une nouvelle responsabilité relative au suivi de l'argent public dans les organisations privées. Ce qui est particulier, c'est le fait que le BVG n'ait jamais été demandeur d'une telle responsabilité qu'il ne compte d'ailleurs pas exercer. Ceci va l'a l'encontre de la théorie d'Abbott qui attribue le pouvoir d'initiative aux groupes revendicateurs.

\section{- CONCLUSION}

Par rapport à la théorie de la société de l'audit, la valeur ajoutée de celle d'Abbott réside dans la mise en évidence du caractère universel des mécanismes mis en lumière par Michael Power : oui, il existe un écart entre le savoir abstrait et les pratiques concrètes effectuées en son nom; oui, cette plasticité du savoir abstrait permet aux groupes qui les maîtrisent de s'approprier certaines activités au détriment d'autres groupes; non, les auditeurs ne sont pas les seuls à être en mesure de tirer profit de ces mécanismes. Chaque groupe manœuvre avec pour objectif de s'approprier une plus grosse part du gâteau que son adversaire, ce gâteau étant l'ensemble du travail disponible au sein du gouvernement. Le script est désormais connu.

Premièrement, le monde universitaire construit constamment de nouveaux savoirs abstraits potentiellement mobilisables par des groupes potentiellement professionnels pour s'attacher un travail gouvernemental : la doctrine de la responsabilité ministérielle est l'un de ces savoirs, comme la rationalisation des choix budgétaires, la vérification de gestion, la vérification interne, la gestion des risques... La liste des savoirs abstraits possédant quelque application possible dans le secteur public est pratiquement infinie et s'enrichit tous les jours de nouveaux arrivants.

Deuxièmement, une organisation (publique dans le cas qui nous occupe) se saisit d'un de ces savoirs disponibles sur le marché intellectuel et en vient à (re)définir son identité, sa mission, selon ces termes : le SCT troque son costume d'agent du budget pour celui de conseil de gestion du gouvernement; le BVG, celui d'officier du Parlement pour celui de vérificateur compréhensif. Le choix du savoir abstrait se fait en fonction des circonstances et des possibilités qu'il laisse entrevoir. Par exemple, l'identité de conseil de gestion du gouvernement n'apparaît pertinente au SCT que dans la mesure où il est en train de perdre ses dernières compétences relatives à l'allocation des ressources.

Enfin, les organisations publiques potentiellement professionnelles expriment par ce savoir abstrait une revendication formelle d'expertise exclusive sur un problème particulier : dès lors que nous sommes les professionnels qui de l'audit qui du budget, et que la zone projetée par cette expertise couvre d'autres zones de tâche que nous ne pouvons encore effectuer, nous réclamons le droit exclusif de 
professer sur ces zones de tâche. Dans le cas de l'auditoire public, les hommes politiques sont soit tellement aveuglés par les avantages potentiels que leur font miroiter les revendications professionnelles (rationalisation des choix budgétaires : le budget n'est plus l'objet de luttes politiques; BCG 2.0 : tout est sous contrôle) soit motivés par des objectifs stratégiques (BCG 1.0 : sauvegardons le SCT) qu'ils acceptent généralement sans grandes difficultés de céder une partie substantielle de leur souveraineté à un savoir abstrait et à son dépositaire institutionnel. Le cas de la responsabilité de vérification de gestion du BVG constitue à cet égard une exception, bien qu'il puisse être soutenu que le gouvernement a alors préféré de faibles bénéfices immédiats (l'absence d'opprobre du public) à de grands bénéfices futurs (le maintien de cette zone de tâche dans son giron).

Sans vouloir minimiser l'immense défi que représente la transformation d'une administration bureaucratique en une organisation professionnelle (il a fallu plus de trente ans au BVG pour effectuer sa métamorphose), l'obtention par une administration publique d'une responsabilité professionnelle n'est pas insurmontable : au moins cinq cas ont pu être recensés en quarante ans d'histoire canadienne dans la zone de tâche considérée. Pourtant, les réelles difficultés ne se sont fait sentir qu'à ce moment, c'est-à-dire lorsqu'il s'agit de réaliser sur le lieu de travail les formidables attentes suscitées afin d'obtenir la juridiction - un aspect presque entièrement ignoré par la théorie de la société de l'audit, sous prétexte que l'écart entre savoir abstrait et pratiques concrètes est invisible de l'extérieur.

En réalité, les contraintes nécessaires à l'obtention d'une juridiction portent en elles les germes de sa disparition. Pour convaincre l'auditoire étatique d'attribuer une juridiction, un groupe potentiellement professionnel peut être enclin à promettre plus de bénéfices que son savoir abstrait n'est vraisemblablement en mesure d'apporter; pour préserver une juridiction, il s'agirait de ne promettre que ce que le savoir abstrait est susceptible d'apporter réellement. Mais personne n'obtient de juridiction de cette façon.

Ce problème est amplifié par la tendance structurelle des groupes administratifs potentiellement professionnels à revendiquer des juridictions dont ils délègueraient l'application aux ministères : il est plus simple de réclamer aux ministères la production d'études d'efficacité pour appuyer les décisions budgétaires (rationalisation des choix budgétaires) que de cibler soi-même scientifiquement les programmes devant être financés ou supprimés. Ces groupes professionnels comptent généralement sur la persuasion (il est souvent question de changement culturel, d'adoption d'une mentalité d'analyse des risques...) pour traduire leur juridiction légale sur le lieu de travail. Mais ce travail de conquête doit être réitéré sur le lieu de travail, et les revendications émanant de l'auditoire étatique se heurtent souvent à des occupants forts - les gestionnaires - peu enclins à céder une partie de leur travail à un autre savoir abstrait que le leur. Cette combinaison d'incitants à promettre plus que l'on ne peut réellement donner (afin d'obtenir une juridiction) et de "désincitants " à ne promettre que ce que l'on peut donner (car ce défi incombera à autrui) fait en sorte que la plupart des juridictions professionnelles sont mort-nées. 
Mais comment la vérification de gestion a-t-elle pu demeurer du ressort du BVG pendant plus de quarante-cinq ans? Parallèlement à cette question se pose évidemment celle de savoir si la compétition professionnelle est bel et bien libre, comme le sous-entend Abbott, ou si elle est faussée, comme le laisse entendre Power.

Premièrement, le BVG effectue seul le travail qu'il conçoit : il n'est pas tributaire du bon vouloir de gestionnaires de ratifier sa juridiction sur le lieu de travail. Il est permis à tout groupe potentiellement professionnel de souhaiter effectuer son travail seul, même si beaucoup ne le font pas.

Deuxièmement, le BVG dispose non seulement d'une juridiction étatique, mais aussi d'une juridiction publique sur la performance administrative : tant qu'il détient fortement cette juridiction publique, le gouvernement n'est pas réellement en mesure de lui retirer sa juridiction étatique, à moins de s'attirer les foudres de l'opinion publique et de menacer sa réélection. À nouveau, libre par exemple au BCG de projeter sa juridiction étatique sur la place publique en publiant ses rapports, ce que le BVG, fort de son expérience en la matière, lui recommande d'ailleurs vivement (OAG, 2011).

Enfin, il est vrai que le BVG possède un avantage concurrentiel sur ses adversaires. Son pouvoir de vérification de gestion consiste précisément à rendre visible l'échec (ou le succès) d'autres entités, et l'administration publique de cette preuve a été déterminante dans plusieurs luttes juridictionnelles : la création du BCG ("Le Parlement a perdu la maîtrise des fonds publics »-BVG dans Sinclair, 1979, p. 143), sa réintégration dans le SCT (" la vérification interne a échoué à se montrer digne des attentes à être une fonction vibrante »-OAG, 1993), et sa récente résurrection (" les hauts fonctionnaires ont enfreint toutes les règles"-OAG, 2002). Comme la juridiction du BVG est exclusive et qu'il est formellement indépendant, aucun concurrent n'est en mesure d'établir son échec; Michael Power marque un point.

Toutefois, la position du BVG semble de plus en plus menacée. D’une part, la comparution de la vérificatrice générale Sheila Fraser devant la commission Gomery aurait pu tourner plus mal : il aurait pu être publiquement établi que le BVG n'eut pas appliqué son savoir abstrait de manière à ce que deux auditeurs arrivent aux mêmes conclusions (voir Fraser, 2007) et le BVG eut réagi en renforçant la formalisation de ses pratiques professionnelles (interview avec un cadre du BVG, 2009). D'autre part, l'invitée surprise de la Loi fédérale sur la responsabilité $^{4}$ - la soumission des organisations privées recevant des subsides fédéraux aux juridictions du BVG - a l'apparence d'un cadeau empoisonné pour le BVG. À ressources égales, soit il effectue ce travail au risque d'avoir moins de ressources à investir dans la fourniture de munitions à l'opposition et aux médias pour attaquer la majorité (Malloy, 2004), soit il ne l'effectue pas - ce qui est l'option défendue actuellement par le BVG (interview avec un cadre du BVG, 2009). Si toutefois un

\footnotetext{
${ }^{4}$ La Loi fédérale sur la responsabilité contient d'autres dispositions potentiellement menaçantes pour le BVG, notamment l'instauration du Directeur parlementaire du budget. À l'époque où les données empiriques ont été recueillies, les contours précis de ce nouvel acteur n'étaient pas suffisamment clairs pour être inclus dans l'analyse. S'il était en mesure de développer une juridiction propre sur son travail, ce nouvel acteur pourrait devenir à terme un concurrent du BVG. À l'époque toutefois, le BVG excluait cette possibilité (interview avec un cadre du BVG, 2009).
} 
scandale éclatait impliquant une organisation privée soumise théoriquement au contrôle du BVG, celui-ci aurait du mal a éviter d'endosser la responsabilité que le gouvernement n'hésiterait pas à lui faire porter. Dans les deux cas de figure, ce mandat menace le BVG et ses juridictions.

Plus que l'Arlésienne de la société de l'audit ou un retour en grâce du politique, la tendance à plus long terme que nous laisse entrevoir la théorie d'Abbott est celle d'un rééquilibrage progressif des pouvoirs professionnels au sein du gouvernement fédéral canadien, avec un BVG qui pourrait bien avoir atteint le faîte de son pouvoir au début de septembre 2004 devant le juge John Gomery (2004a, 2004b et 2004c), un BCG qui semble avoir tiré certaines leçons des erreurs du passé (Larson et Zussman, 2010) et un SCT qui bon an mal an occupe son rôle de gestionnaire du gouvernement depuis presque vingt ans.

Il n'y a donc pas de société de l'audit au Canada, mais un système des professions au sein du gouvernement fédéral. Certes, les maux attribués à la société de l'audit pourraient alors être reportés sur ce système professionnel qui promet plus qu'il ne peut donner, qui reste " fondamentalement obscur " et qui usurpe en dernière analyse le pouvoir démocratique légitime des élus. Toutefois, si la démocratie n'est pas comprise dans le sens de Rousseau comme le gouvernement par le peuple, mais dans celui de Montesquieu comme l'existence de contre-pouvoirs, l'émergence d'un contre-pouvoir professionnel au pouvoir politique peut être perçue comme servant plutôt que desservant la démocratie. Et c'est en permettant l'adoption de ce regard froid - peut-être légèrement cynique - loin des discours enflammés pour ou contre la société de l'audit, que la théorie d'Abbott révèle toute sa pertinence.

\section{BIBLIOGRAPHIE}

Abbott, A. (2005). "Linked Ecologies: States and Universities as Environments for Professions ", Sociological Theory, vol. 23, n 3, p. 245-274.

Abbott, A. (2003). "Écologies liées : à propos du système des professions ", dans P.-M. Menger, Les professions et leurs analyses sociologiques, Paris, INED, p. 29-50.

Abbott, A. (1988). The System of Professions: An Essay on the Division of Expert Labour, Chicago, The University of Chicago Press.

Benoit, L. E. et C. E. S. Franks (2006). « Faute d'un clou : le rôle de la vérification interne dans le scandale des commandites ", Rétablir l'imputabilité, études vol. 2, Ottawa, Travaux publics et Services gouvernementaux, p. 261-337.

Brophy, J. et L. M. McGimpsey (1980). «Internal Audit and Program Evaluation in the Government of Canada ", Optimum, vol. 11, n² 2, p. 16-25.

Champy, F. (2009). La sociologie des professions, Paris, Quadrige/PUF.

Clark, I. D. et H. Swain (2005). « Distinguishing the Real from the Surreal in Management Reform: Suggestions for Beleaguered Administrators in the Government of Canada ", Canadian Public Administration, vol. 48, $n^{\circ}$ 4, p. 453-476.

Cutt, J. (1988). Comprehensive Auditing in Canada: Theory and Practice, New York, Praeger.

Dubar, C. et P. Tripier (1998). Sociologie des professions, Paris, Armand Collin. 
Francis, J. R. (1994). « Auditing, Hermeneutics, and Subjectivity », Accounting, Organizations and Society, vol. 19, n 3, p. 235-269.

Fraser, B. W. (2004). " Cool under Fire », Internal Auditor, vol. 61, n 4, p. 72-77.

Fraser, S. (2007). "Public Scrutiny Puts SAI Audit Practices to the Test ", International Journal of Government Auditing, $\mathrm{n}^{\circ}$ 1, p. 1-5.

Free, C. et V. Radcliffe (2009). " Accountability in Crisis: The Sponsorship Scandal and the Office of the Comptroller General in Canada ", Journal of Business Ethics, vol. 84, $\mathrm{n}^{\circ} 2$, p. 189-208.

Glynn, J. J. (1985). "Value for Money Auditing: An International Review and Comparison ", Financial Accountability \& Management, vol. 1, n² 2, p. 113-128.

Gomery, J. H. (2004a). Audience publique / Public Hearing, vol. 1, Ottawa, Commission d'enquête sur le programme de commandites et les activités publicitaires, 7 septembre.

Gomery, J. H. (2004b). Audience publique / Public Hearing, vol. 2, Ottawa, Commission d'enquête sur le programme de commandites et les activités publicitaires, 8 septembre.

Gomery, J. H. (2004c). Audience publique / Public Hearing, vol. 3, Ottawa, Commission d'enquête sur le programme de commandites et les activités publicitaires, 9 septembre.

Gomery, J. H. (2004d). Audience publique / Public Hearing, vol. 4, Ottawa, Commission d'enquête sur le programme de commandites et les activités publicitaires, 13 septembre.

Good, D. A. (2007). Politics of Public Money: Spenders, Guardians, Priority Setters, and Financial Watchdogs inside the Canadian Government, Toronto, University of Toronto Press.

Harder, P. et E. Lindquist (1997). "Expenditure Reporting and Reporting in the Government of Canada: Recent Developments and Backgrounds ", dans J. Bougault, M. Demers et C. Williams, Public Administration and Public Management: Experiences in Canada, Québec, Publications du Québec, p. 71-89.

Hodgetts, J. E. (2007). " Royal Commissions and Public-service Reform: Personal Reflections ", Canadian Accounting Perspectives, vol. 50, n 4, p. 525-540.

Hollander, M. J. et M. J. Prince (1993). « Analytical Units in Federal and Provincial Governments: Origins, Functions and Suggestions for Effectiveness ", Canadian Public Administration, vol. 6, n 2, p. 190-224.

Johnson, A. W. (1971). «The Treasury Board of Canada and the Machinery of Government of the 1970s ", Revue canadienne de science politique, vol. 4, n 3, p. 346-366.

Johnson, D. (2002). Thinking Government: Ideas, Policies, Institutions and Public-Sector Management in Canada, Petersborough, Broadview Press Ltd.

Kelly, J. et E. Lindquist (2003). « Metamorphosis in Kafka's Castle: The Changing Balance of Power among the Central Budget Agencies of Canada ", dans J. Wanna, L. Jensen et J. De Vries, Controlling Public Expenditure, Bodmin, Edward Elgar, p. 85-105.

Larson, M. S. (1977). The Rise of Professionalism: A Sociological Analysis, Berkeley, University of California Press.

Larson, P. et D. Zussman (2010). " Departmental Audit Committees: An Evaluation », Optimum, vol. 40, n 4, p. 29-50. 
Malloy, J. (2004). «An Auditor's Best Friend? Standing Committees on Public Accounts ", Canadian Public Administration, vol. 47, n 2, p. 165-183.

O’Neal, B. (1994). Reorganizing Government: New Approaches to Public Service Reform, Ottawa, Library of Parliament.

OAG (2011). 2011 June Report of the Auditor General of Canada, Ottawa, Office of the Auditor General of Canada.

OAG (2004). 2004 November Report of the Auditor General of Canada, Ottawa, Office of the Auditor General of Canada.

OAG (2002). 2002 May Report of the Auditor General of Canada, Ottawa, Office of the Auditor General of Canada.

OAG (1993). 1993 Report of the Auditor General of Canada, Ottawa, Office of the Auditor General of Canada.

OAG (1987). 1987 Report of the Auditor General of Canada, Ottawa, Office of the Auditor General of Canada.

OAG (1984). 1984 Report of the Auditor General of Canada, Ottawa, Office of the Auditor General of Canada.

OAG (1983). 1983 Report of the Auditor General of Canada, Ottawa, Office of the Auditor General of Canada.

Paquin, M. (1997). "Administrative Modernization within the Canadian Government", J. Bourgault, M. Demers et C. Williams, Public Administration and Public Management Experiences in Canada, Québec, Publications du Québec, p. 143-154.

Power, M. (1999). The Audit Society: Rituals of Verification, Oxford, Oxford University Press.

Prince, M. J. et J. A. Chenier (1980). « The Rise and Fall of Policy Planning and Research Units: An Organizational Perspective ", Canadian Public Administration, vol. 23, n 4, p. 519-541.

Rogers, H. (1978). " Management Control in the Public Service ", Optimum, vol. 9, n 3, p. $14-28$.

Rowat, D. C. (1963). " Canada's Royal Commission on Government Organization ", Public Administration, vol. 41, $\mathrm{n}^{\circ}$ 2, p. 193-205.

Saint-Martin, D. (1998). «The New Managerialism and the Policy Influence of Consultants in Government: An Historical-Institutionalist Analysis of Britain, Canada and France ", Governance: An International Journal of Policy, Administration and Institutions, vol. 11, n³, p. 319-356.

Savoie, D. (2008). Court Government and the Collapse of Accountability in Canada and the United Kingdom, Toronto, University of Toronto Press.

Savoie, D. (1996). « Reforming the Expenditure Budget Process: The Canadian Experience ", dans D. J. Savoie, Budgeting and the Management of Public Spending, Cheltenham, Brookfield, p. 299-314.

Sinclair, S. (1979). Cordial but not Cosy: A History of the Office of the Auditor General, Toronto, McCelland and Stewart.

Sutherland, S. L. (2002). The Office of the Auditor General of Canada: Government in Exile?, Working Paper no 31, p. 1-29.

Sutherland, S. L. (1991). « Responsible Government and Ministerial Responsibility: Every Reform Is Its Own Problem ", Revue canadienne de science politique, vol. 24, n 1, p. 91-120. 
Sutherland, S. L. (1990). "The Evolution of Program Budget Ideas in Canada: Does Parliament Benefit from Estimates Reform? ", Canadian Public Administration, vol. 33, $\mathrm{n}^{\circ} 2$, p. 133-164.

Sutherland, S. L. (1986). «The Politics of Audit: The Federal Office of the Auditor General in Comparative Perspective ", Canadian Public Administration, vol. 29, n 1, p. 118-148.

Sutherland, S. L. (1981). "State Audit: A Canadian Maverick? », Public Money, vol. 1, n³ 3 , p. 39-43.

Sutherland, S. L. (1980). "On the Audit Trail of the Auditor General: Parliament's Servant 1973-1980 ", Canadian Public Administration, vol. 23, n 4, p. 616-644.

Troupin, S. (2012). Professionalizing Public Administration(?) The Cases of Performance Audit in Canada and the Netherlands, Thèse défendue en vue de l'octroi du titre de $\mathrm{PhD}, \mathrm{KU}$ Leuven. 